\title{
Ethnicity, socioeconomic position and severity of problems as predictors of mental health care use in 5- to 8-year-old children with problem behaviour.
}

Bevaart F \& Mieloo CL, Wierdsma A, Donker MC, Jansen W, Raat H, Verhulst FC, van Oort FV.

Soc Psychiatry Psychiatr Epidemiol. 2014 May;49(5)

F. Bevaart and C.L. Mieloo are both first co-authors on this article. 


\begin{abstract}
Background

Empirical research on mental health care use and its determinants in young school-aged children is still scarce. In this study, we investigated the role of ethnicity, socioeconomic position (SEP) and perceived severity by both parents and teachers on mental health care use in 5-8-year-old children with emotional and/or behavioural problems.
\end{abstract}

Methods

Data from 1,269 children with a high score (>P90) on the Strengths and Difficulties Questionnaire (SDQ) in the school year 2008-2009, were linked to psychiatric case register data over the years 2010-2011. Cox proportional hazards models were used to predict mental health care use from ethnicity, SEP and perceived severity of the child's problems.

Results

During the follow-up period 117 children with high SDQ scores $(9.2 \%)$ had used mental health care for the first time. Ethnic minority children were less likely to receive care than Dutch children (HR Moroccan/Turkish: $0.26 ; 95 \% \mathrm{Cl}: 0.13-0.54, \mathrm{HR}$ other ethnicity: $0.26 ; 95 \% \mathrm{Cl}: 0.12-0.58)$. No socioeconomic differences were found. After correction for previous care use, ethnicity and parental perceived severity, impact score as reported by teachers was significantly associated with mental health care use (HR: $1.58 ; 95 \% \mathrm{Cl}: 1.01-2.46)$.

\title{
Conclusions
}

Ethnicity is an important predictor of mental health care use in young children. Already in the youngest school-aged children, ethnic differences in the use of mental health care are present. A distinct predictor of care use in this age group is severity of emotional and behavioural problems as perceived by teachers. Therefore, teachers may be especially helpful in the process of identifying young children who need specialist mental health care. 


\section{Introduction}

Detection and treatment of emotional and behavioural problems at an early age is becoming more and more important [1], especially since we know that these problems can negatively influence children's daily lives [2] and tend to be persistent if left untreated [3, 4]. Therefore, the development of empirically informed public policies with regard to a proper response to such problems in young children is a priority on the global health agenda $[5,6]$. One way of treating emotional and behavioural problems in young children is through offering specialist mental health care. However, research on exact rates and characteristics of mental health care use in the youngest school-aged children (5-8 years old) is still scarce.

Rates of mental health care use in children differ within and across countries $[7,8]$, since service organisation and availability of services differ greatly [9]. In Western countries, between $5 \%$ and $21 \%$ of all children in the community have used some sort of service for mental health problems in the previous year [8]. Rates of specialist mental health treatment for children across countries are lower: up to $8 \%$ of all children have used these services [8]. Most of the large community studies on child psychopathology and care use, however, do not include the youngest school-aged children [7]. The Great Smoky Mountains study and the Methodology for Epidemiology in Children and Adolescents study (MECA), for example, both begin at age $9[9,10]$. A rare exception is a study in the United States that described mental health care use in a nationally representative sample including 3-5-year-old children as well as older children [11].

Studying the characteristics of children receiving mental health care is complex, since there are large differences in sample selection, diagnostic criteria, definition of service use and age range across studies [12]. Nevertheless, studying these characteristics is important, since differences in mental health care use across ethnicities and socioeconomic position groups have been consistently reported in older children [7, 8, 13]. A review by Flores et al. (2010) indicated lower service use and under-treatment for ADHD in ethnic minority groups [14]. Other studies examining ethnicity as predictor of service use in children, however, show both over- and underrepresentation of mental health care use in ethnic minority groups [7]. The association between gender and service use seems quite clear throughout literature: several studies indicated female gender being a strong barrier to mental health service use $[15,16]$; one recent study showed that, after controlling for the number of problem areas, boys were twice as likely as 
girls to be in contact with a mental health care service [17]. The association between socioeconomic position (SEP) and service use is somewhat more contradictory throughout literature. Whereas some studies reported no associations with SEP $[8,18]$, other studies reported that low SEP was slightly associated with more service use [19-21]. Furthermore, other research indicates greatest use in middle or high socioeconomic position groups $[22,23]$. These findings may reflect differences in care use and accessibility across countries [8], or may reflect methodological differences between studies, like sample size and different definitions of SEP. Finally, severity of emotional and behavioural problems is one of the most important predictors of service use [24-27]. Most studies that use questionnaires to measure emotional and behavioural problems, suggest that total symptom scores and impairment ratings are robust predictors of mental health care use [26].

Describing mental health care use and its determinants is especially important in the group of the youngest school-aged children. For these children a major milestone takes place; the transition from preschool to elementary school. Children must accommodate to daily schedules, new adult authority, peers and academic challenges through which emotional and behavioural problems can become apparent [28]. To detect such problems in this age group, both parent and teacher report of problems are important, since inter-rater differences in level of problems are reported frequently [29, 30]. These differences may have a significant impact on the identification and treatment of mental health problems in children [29]. Inter-rater differences can reflect both perceptual bias [31], or true differences in the problem behaviour of children across settings [32]. In the Netherlands, signalling for emotional and behavioural problems is part of the preventive care system and is based on both parent and teacher report. Although signalling is frequently conducted in the youngest school-aged children, exact numbers on mental health care use after signalling are still lacking.

Hence, the main aim of this study was to describe specialist mental health care use in children of 5-8 years old with high scores for emotional and behavioural problems and to examine whether SEP and ethnicity were related to the likelihood of receiving mental health care. In a group of 1,269 children, with a high score (>P90) on the Strengths and Difficulties Questionnaire (SDQ) at age 5-6 years, we described service use during 24 months following signalling. The second goal of this study was to test whether both parental perceived severity of emotional and behavioural problems and perceived severity by teachers are predictive of mental health care use. 


\section{Methods}

\section{Setting}

In the Netherlands, the Dutch preventive care system is responsible for monitoring and safeguarding the development of all children, for example through identification of children with emotional and/or behavioural problems with signalling questionnaires. The Dutch preventive care system offers publicly funded preventive programs for all children from birth to 19 years. As part of this system, more than $90 \%$ of all children undergo 3 to 4 routine health assessments by a CHP during their school careers; the first in grade two of primary school (mean age: 5-6-years). Screening questionnaires, including the Strengths and Difficulties Questionnaire (SDQ) [33-36], are used to identify children with emotional and/or behavioural problems before the appointment with the CHP. During the health assessment the CHP discusses psychosocial well-being of the child with the parents. The CHP can give parents of children with problem behaviour advice or reassurance, the CHP can make a new appointment with the parents and the child for further diagnosis and/or counselling, or the CHP can refer these children to professional care. Children can be referred to preventive care (for example to enhance parenting skills), to specialized youth social work or child protective services, to the child mental health system or to other sources of care (e.g. care provided by school). Child mental health care in the Netherlands comprises institutes for ambulatory mental health care, and psychiatric outpatient and inpatient clinics, which provide diagnostic assessment, treatment and assistance to children and their caregivers.

\section{Subjects}

In the school year 2008-2009, from a total of 11,987 children enrolled in grade two (5-6 years old) of $94 \%$ of all mainstream elementary schools in the Rotterdam-Rijnmond area, the SDQ was completed for 10,951 (91\%) children. Questionnaires were distributed through schools to parents and teachers for use as a signalling tool in the preventive child health care. In total, for $8,114(67 \%)$ children parents filled out the questionnaire and in total for $9,397(80 \%)$ children teachers filled out the questionnaire. A total of 1,552 children had a high SDQ total difficulties score reported by parent, teacher or both. A high SDQ total score was defined as a score above the $90^{\text {th }}$ percentile $(>P 90)$ in the total group of 10,951 children. This cut-off reflects children with both clinical and subclinical levels of problem behaviour [37] and is suggested 
by the test developers (www.sdq-info.org). The cut-off point in this population was 14 for parents and 13 for teachers.

In the group of 1,552 children with a high SDQ score, parental consent for linkage was refused for 34 children (2\%) and 248 children (16\%) could not be linked to the Psychiatric Case Register (PCR) for various reasons, among which: the SDQ was completed after the start of the follow-up period, children moved outside the Rotterdam-Rijnmond area, or children missed required identifiers for the linkage. In total, data on mental health care use was available for 1,269 children ( $82 \%$ of the target population). Nonresponse was higher among female children $(p=0.004)$, among children living in a family composition other than both biological parents $(p=0.005)$, and among children with a low severity perception according to their parents $(p<0.001)$ and teachers $(p<0.001)$.

Informed consent was obtained from parents in the study population. This study was approved by the Medical Ethics Committee of the Erasmus University Medical Centre Rotterdam, the Netherlands. This study was conducted according to the Declaration of Helsinki code of ethics.

\section{Measures}

Problem behaviour and severity: The Strengths and Difficulties Questionnaire (SDQ) was used to obtain standardised reports of children's problem behaviour and problem severity, as reported by parents and teachers. The SDQ is a reliable and valid 25 -item measure to identify 3-16-year-old children with emotional and behavioural problems [33-36]. The SDQ measures conduct problems, inattentionhyperactivity, emotional problems, peer problems and prosocial behaviour and impact of the problems. Goodman's impact supplement [38] starts with an item about perceived problems and if confirmed, it follows with items about distress, social impairment, burden and chronicity. The impact score ranges from 0-10 for parents and 0-6 for teachers. A high impact score was defined as having a score of two or higher on the impact questions [38]. SDQ total score and SDQ impact score were used as separate indicators of severity.

Demographic variables: Socioeconomic position (SEP) of the parents and ethnicity of the child were included as predictors. Gender of the child and previous use of care were included as confounders. Indicators of SEP were parental level of education and average neighbourhood family income. The level of education of the parents was classified into three levels, ranging from 1 (low) to 3 (high). A low education 
was defined as no education at all, or only elementary school. A high education was defined as higher vocational education or university degree. Average neighbourhood family income (further referred to as family income), based on the six-digit postal code system as used in the Netherlands, was obtained from Statistics Netherlands (CBS, 2004). Family income was classified in three groups: low, middle and high. A low family income was defined as equal as or lower than the established minimum income in the Netherlands (<€1401 per month). A high family income was defined as equal as or higher than the established modal income in the Netherlands (>€ 2508 per month).

A child was classified as ethnic Dutch, Surinamese, Antillean, Turkish, Moroccan or other, based on the country of birth of the child and at least one of his/her parents [39]. If the country of birth of one of the parents, or the child itself was outside the Netherlands, the child was classified as non-Dutch [39]. The children were divided in four groups: Dutch, Antillean/Surinamese (children from former colony migrants), Moroccan/Turkish (children from labour migrants) and children with other ethnicities. The largest ethnic minority groups living in the Netherlands migrated from Mediterranean countries, mainly Turkey and Morocco, as labour migrants since the 1960s and early 1970s. Surinamese and Antillean migrants came from South America and the Caribbean respectively, to the Netherlands during the process of decolonisation after 1975 . The group containing other ethnicities is very diverse, since this group is comprised of, among others, labour migrants, refugees and knowledge migrants.

Use of mental health care: Most studies of mental health care in children use self-report of children and/or parents, which can be biased by recall problems or unwillingness to report referral. Therefore, in this study data on the use of child and adolescent mental health services $(\mathrm{CAMH})$ were obtained from the Psychiatric Case Register Rotterdam-Rijnmond. A psychiatric case register is a "patient-centred longitudinal record of contacts with a defined set of psychiatric services, originating from a defined population" [40]. This prevents patients from being counted more than once, while at the same time keeping track of all patient contacts over time. The Psychiatric Case Register Rotterdam-Rijnmond contains information on all mental health care services in the area: the Regional Institutes for Outpatient Mental Health Care, other outpatient services and clinics for psychiatric care, crisis intervention services, sheltered homes, day centres and (general) psychiatric hospitals. A negligible proportion of the children and adolescents in care in the Netherlands use other mental health services, e.g. mental health care 
programs provided by the private sector. In most cases these patients have consulted the public sector first [41].

The register data were linked to the children with high SDQ scores using the probabilistic linkage method [42], including the first two letters of the last name, date of birth, gender, country of birth, and partial postal code as identifiers. Anonymity of the subjects was adequately protected, since probabilistic record linkage is based on data that are not traceable to individual patients [42]. The case register provided information on the starting date of treatment. Information on both previous and new service use was obtained from the Psychiatric Case Register (PCR). Previous service use was defined as one or more contacts with the mental health care system before or during signalling problems with the SDQ. New service use was defined as at least one contact with a mental health care service in the follow-up period, after signalling emotional and behavioural problems. Use of mental health services was assessed for a follow-up period of 24 months after signalling. The follow-up period was defined as the length of time (days) between completing the SDQ by parents or teachers and the first contact with mental health services of the child. Mean length of the follow-up period was 696.6 days (SD: 121.2, range: 6.0-730.0). Mean age at the end of the follow-up was 7.6 years (SD: 0.6 ); $8 \%$ was 6 years old or younger, $64 \%$ was 7 years old, $27 \%$ was 8 years old and $1 \%$ was 9 years old.

\section{Data analyses}

Cox continuous-time proportional hazards models were used to test demographic and severity variables as predictors of mental health service use. "Survival time" was defined as the length of time (days) between completing the SDQ and the first contact with mental health services. All Cox regression analyses were adjusted for service use prior to signalling problems with the SDQ (previous service use). We applied a stepwise approach using likelihood-ratio tests to assess significant change in model deviance. Model fit was checked with observed versus expected plots assessing the proportional hazard assumption and by testing the correlation of Schoenfeld residuals for each predictor with survival time.

A significance level of $<.05$ was used for all analyses. To account for missing values we used multiple imputation based on twenty imputed data sets ('multiple imputation' procedure in SPSS 17.0). In the analyses on parent reports we only included children with parental response on the questionnaire and in the analyses on teacher reports we included only children with teacher response. The data were 
imputed only for general characteristics and socioeconomic indicators, but not for severity indicators and mental health care use. Statistical analyses were performed using Statistical Package of Social Sciences, version 17.0 for Windows (SPSS Inc, Chicago, IL, USA).

\section{Results}

Table 1 shows the characteristics of the study population. Mean age of children at baseline was 5.3 (SD: 0.5) years and $64.2 \%$ of the children were male. Mean parent SDQ score was 14.3 (SD: 5.1 ) and mean teacher SDQ score was 12.3 (SD: 6.3). Correlation between the parent SDQ total score and the teacher SDQ total score was low (Pearson $r=-0.38, p<0.001$ ). The minority of the children had a high impact score according to parents $(20.7 \%)$, or according to teachers $(31.1 \%)$. Most children in the study sample were Dutch (36.8\%), had parents with a middle educational level (57.6\%) and a middle income (43.6\%).

In the study sample $(n=1,269) 97(7.6 \%)$ children received care before the initial signalling with the SDQ (previous care use). During the follow-up period, 117 (9.2\%) children had newly entered the Psychiatric Case Register, indicating that they had at least one contact with mental health service in the region. Mean survival time was 696.61 days (SD 121.21; min. 6; max. 730). 
Table 1 Characteristics of the study population $(n=1269)$

No. Percentage/mean (SD)

Gender of the child

Female

454

$35.8 \%$

Male

815

$64.2 \%$

Ethnicity of the child

Dutch 468

$36.8 \%$

Antillean/Surinamese 231

$18.2 \%$

Moroccan/Turkish $\quad 354$

$27.9 \%$

Other

217

$17.1 \%$

Parental education level

High

361

Middle

731

$28.4 \%$

Low

176

$57.6 \%$

$13.9 \%$

Family income

Continuous

High (above $>€ 2508$ )

251

Middle $(€ 1401-€ 2508)$

Low $(<€ 1401)$

464

$1842(697)$

$19.8 \%$

$43.6 \%$

$36.6 \%$

Mean age of the child at start

$5.3(0.5)$

(SD)

Mean age of the child at end

follow up (SD)

Mean follow up period in days

(SD)

\section{Parent SDQ report}

Total score (mean (SD))

$$
\text { Low }
$$

High

286

682

Impact score

Low

716

High

187

Teacher SDQ report

Total score (mean)

High $\quad 722$

Impact score (mean)

781

High

395

$7.6(0.6)$

$696.6(121.2)$

$14.3(5.1)$

$29.5 \%$

$70.5 \%$

$0.82(1.54)$

$79.3 \%$

$20.7 \%$

$12.3(6.3)$

$39.1 \%$

$60.9 \%$

$1.2(1.4)$

$66.4 \%$

$31.1 \%$

Service use in follow up period

117

$9.2 \%$

Service before start follow up

97

$7.6 \%$ 
Table 2 shows the service use by ethnicity, SEP measures and severity, adjusted only for previous service use. Child ethnicity was significantly related to new mental health care use. Mental health care use was lower in non-Dutch children than in Dutch children: $7.4 \%$ in Antillean/Surinamese children, $3.4 \%$ in Moroccan/Turkish children, $5.5 \%$ in children with other ethnicity versus $16.2 \%$ in Dutch children (HR Antillean/Surinamese: 0.39 , HR Moroccan/Turkish: $0.18, \mathrm{HR}$ other ethnicity: 0.30 ). Severity of the problems, in terms of total difficulties score and impact score, was related to a higher chance of service use (HR total score parents: 1.06, HR total score teachers: 1.05, HR impact score parents: 2.15 and HR impact score teachers: 1.85). Parental educational level and family income were not related to service use.

Table 2 Hazard ratios between predictor variables and service use ${ }^{\dagger}$

$\begin{array}{llllll}\text { Percentage } & \mathrm{HR} & 95 \% \mathrm{Cl} & \mathbf{p} & \boldsymbol{\beta} & \mathrm{SE}\end{array}$

\section{Gender of the child}

$\begin{array}{lllllll}\begin{array}{l}\text { Female } \\ \text { Male }\end{array} & 4.6 \% & 1.00 & & & & \\ \text { Mutch of the child } & 11.8 \% & 2.68^{*} & 1.67-4.30 & <0.001 & 0.99 & 0.24 \\ \begin{array}{lllllll}\text { Dutch } \\ \text { Antllean/Surinamese }\end{array} & 16.2 \% & 1.00 & & & & \\ \text { Moroccan/Turkish } & 7.4 \% & 0.39^{*} & 0.22-0.69 & 0.001 & -0.94 & 0.29 \\ \text { Other } & 3.4 \% & 0.18^{*} & 0.10-0.33 & <0.001 & -1.73 & 0.32 \\ & 5.5 \% & 0.30^{*} & 0.16-0.56 & <0.001 & -1.19 & 0.32\end{array}$

\section{Parental education level}

High

Middle

$\begin{array}{ll}7.5 \% & 1.00\end{array}$

Low

$10.5 \% \quad 1.43$

1.43
0.97

$0.82-2.48$

0.21

0.36

0.28

Family income

High (above $>€ 2508) \quad 11.1 \% \quad 1.00$

Middle (€1401-€2508)

$10.3 \%$

0.93

$0.40-2.34$

0.95

$-0.03$

0.45

Low (<€1401)

$6.9 \%$

0.60

0.58-1.50

0.77

$-0.07$

0.24

0.34-1.06

0.08

$-0.51$

0.29

\section{Parent SDQ report}

Total score (continuous) NA

$1.06^{*}$

$1.01-1.10$

0.009

0.06

0.02

Impact score

$\begin{array}{lllllll}\text { Low } & 8.9 \% & 1.00 & & & & \\ \text { High } & 15.5 \% & 2.15^{\star} & 1.39-3.34 & 0.001 & 0.77 & 0.22\end{array}$

\section{Teacher SDQ report}

Total score (continuous) NA Impact score

$\begin{array}{lll}\text { Low } & 7.4 \% & 1.00 \\ \text { High } & 12.4 \% & 1.85\end{array}$

$\begin{array}{lllll}1.05^{*} & 1.01-1.08 & 0.005 & 0.04 & 0.02\end{array}$

t: all associations are corrected for previous service use. 
${ }^{*}$ : significant at $p<0.05$.

Table 3 shows the results of the multivariate Cox regression model. This model included child gender, previous care, child ethnicity, impact score parent- or impact score teacher, total SDQ difficulties score of the parent report- or total SDQ difficulties score of the teacher report. Inclusion of parental educational level, family income, and impact score of the parent SDQ and impact score of the teacher SDQ did not improve the model significantly. Child ethnicity was still significantly related to new service use. Mental health care use was lower in Moroccan/Turkish children and in children with other ethnicity than in Dutch children. Severity was related to a higher HR for service use for the total SDQ scores rated by parents and teachers. 
Table 3 Hazard ratios of multivariate analyses for ethnicity, problem severity and service use corrected for gender and previous care use

$\begin{array}{llllll}\text { HR } & 95 \% \mathrm{Cl} & \mathbf{p} & \boldsymbol{B} & \text { SE }\end{array}$

Model $1^{\dagger}(n=899)$

Gender of the child

Female $\quad 1.00$

Male $2.08^{\star}$

Ethnicity of the child

Dutch $\quad 1.00$

Antillean/Surinamese 0.67

$\begin{array}{lllll}0.67 & 0.37-1.20 & 0.18 & -0.40 & 0.30\end{array}$

$\begin{array}{llllll}\text { Moroccan/Turkish } & 0.25^{*} & 0.12-0.49 & <0.001 & -1.41 & 0.40\end{array}$

$\begin{array}{llllll}\text { Other } & 0.31^{*} & 0.15-0.66 & 0.002 & -1.16 & 0.38\end{array}$

Parent SDQ report

Total score

1.04

$1.00-2.31 \quad 0.07$

0.04

0.02

Impact score

Low $\quad 1.00$

High $\quad 1.50$

$0.97-2.31 \quad 0.11$

0.40

0.25

Model $2^{\dagger}(n=1088)$

Gender of the child

Female $\quad 1.00$

Male $2.45^{\star}$

Ethnicity of the child

Dutch $\quad 1.00$

Antillean/Surinamese $0.35^{*}$

$\begin{array}{lllll}0.35^{*} & 0.19-0.68 & 0.002 & -1.04 & 0.33\end{array}$

Moroccan/Turkish $\quad 0.19^{\star}$

$\begin{array}{llll}0.10-0.36 & <0.001 & -1.68 & 0.33\end{array}$

Other $\quad 0.30^{*}$

$\begin{array}{llll}0.16-0.58 & <0.001 & -1.21 & 0.34\end{array}$

Teacher SDQ report

Total score $\quad 1.03$

Impact score

Low $\quad 1.00$

High 1.58

$0.99-1.07$

0.11

0.03

0.02

$1.58^{*}$

$1.01-2.46 \quad 0.04$

0.46

0.23

t: Analyses corrected for: gender of the child, previous care, ethnicity of the child, impact score parent/teacher, total difficulties score parent/teacher SDQ report.

*: significant at $p<0.05$

\section{Discussion}

To our knowledge, this study is the first to provide estimates of specialist mental health care use in a population of the youngest school-aged children, after signalling for emotional and behavioural problems through parents and teachers. Overall, the data showed that $9.2 \%$ of children with emotional and behavioural problems receive specialist mental health care within 24 months after signalling and that ethnic minority children are underrepresented in this group. It should be emphasised, however, that the 
results concern numbers of children with high SDQ scores that receive specialist mental health care and do not reflect the total rates of any service use throughout the general population.

\section{Role of ethnicity}

The lower service use in Moroccan and Turkish children and in children from other ethnicities was not related to a lower socioeconomic position or to ethnic variations in the severity of the problems, whereas the lower service use in Antillean and Surinamese children was. It is possible that the migration history of these groups plays a role in these differences. Familiarity with the Dutch way of monitoring and organising care for children may vary with migration factors. For example, Moroccan and Turkish migrants (parents) have had a wider bridge to gap in terms of mastering Dutch language than migrants from former colonies. Besides migration factors, cultural factors may explain ethnic differences in service use. A plausible cultural factor is ethnic differences in care preferences, since there are indications that more ethnic differences in care use exists in specialist care, than in primary care [43]. For example, parents of ethnic minority children may prefer care outside the mental health care system, such as care provided by school or informal care, to solve the problems of their child. Such care preferences may be linked to ethnic differences in parents' attitudes about mental health care services, since these attitudes are associated with whether and which care children receive [44]. Research has suggested that ethnic minority groups have less positive expectations about child mental health services [45].

Furthermore, a clearer understanding is required about predisposing factors and barriers to service use. First of all, it is important to consider that young children are dependent on others, mostly on their parents to access mental health care, to negotiate the system, to make appointments and to pay for their treatment. Characteristics of the family such as a family history of mental illness are associated with a child's chance of receiving specialist mental health care [46]. A study by Farmer et al. (1999) showed that the psychiatric history of a child's primary parent figure was positively associated with persistence and intensity of mental health care use in children [24]. On the other hand, potential barriers can be structural constraints (e.g. lack of availability of providers, long waiting lists), barriers related to perceptions about mental health problems (e.g. parents' and/or teachers' inability to identify children's need for specialist care) and barriers related to perceptions about mental health care services (e.g. lack of trust in mental health care providers). These barriers can be more prevalent in certain ethnic groups than in others, and 
so lead to differences in service use. Whereas one study showed that all three types of aforementioned barriers to mental health care were relatively common among poor urban African-American families in the United States [47], research in other ethnic groups and in other geographic areas is still scarce. More information about the role of barriers to service use in different ethnic groups can aid the development of interventions that address these (potential) barriers. It is important to know which of the suggested barriers are actually perceived as barriers, or act as barriers to service use for young children.

\section{Role of perceived severity}

Perceived severity of the problems according to parent and teacher, were both related to the child receiving specialist mental health care. This extends earlier findings which show that severity of the symptoms perceived by parents, and to a lesser extent by teachers is strongly associated with service use $[8,48-50]$. Interestingly, we showed that the SDQ total score reported by both teachers and parents was related with service use, whereas teacher-reported severity in terms of impact score predicted service use in children stronger than parent-reported impact. Cohen et al. (1991) reported that teachers were the professionals with whom parents most commonly discuss the problems of their child [50] and that thereby teachers can play an important role in initiating children's use of mental health services. Moreover, the Great Smoky Mountains study showed that the education sector was the most common point of entry into mental health care [51]. The association between teacher-reported severity and service use we found in our study was, therefore, not entirely unexpected. This association can be important in the light of low problem perception in parents. The majority of parents of children with high problem scores for emotional and/or behavioural problems, especially ethnic minority parents, do not perceive problems [52], whereas problem perception is an important predictor of service use $[53,54]$. In contrast, teachers' problem perception was much higher than that of parents, and was not related to ethnic background in the same population of young school-aged children [52]. Therefore, teachers' perception of severity of the problems can be important for identifying children that need (professional) care.

The role of teachers in helping children with emotional and behavioural problems should not be limited to detecting problems and initiating specialist care, however. Teachers can also play an important role in facilitating adequate care through school, especially for children with minor problems that do not require specialist care. Although the threshold to use mental health care for young children has lowered, 
many parents still prefer to seek help within a familiar context for their children; teachers, nurses and school psychologists [55]. This could be one of the reasons why approximately $90 \%$ of the children in our study did not receive specialist care. Therefore, more attention should be paid to the possibilities of school-based care and to the training of teachers to enhance their ability to identify and act upon emotional and behavioural problems in young school-aged children. School care can offer parents a low, informal way of dealing with the problems of their child. Furthermore, school care could mitigate the need for future (specialist) intervention.

\section{Role of socioeconomic differences}

Our findings of a lack of socioeconomic differences related to mental health service use are interesting. Mental health care services in the Netherlands seem to be accessible at every socioeconomic level. The results of studies in other European countries with well-developed and easily accessible social and health services are similar to those of the present study $[18,55]$. However, the absence of socioeconomic inequalities in service use cannot be generalised to other countries. Different conclusions are likely to be found in countries where the health care system is organised differently, or in countries where socioeconomic inequalities are larger than in the Netherlands.

\section{Mental health care use in young children}

The interest in early detection of emotional and behavioural problems has increased [1], since several studies have demonstrated the stability of problem behaviour from childhood to adolescence as well as into adulthood [3, 4]. Data collected in studies like this study are sometimes used by those lobbying for services for children [7]. Such mandates are mainly based on the claim that child health care professionals under-identify children with mental health care problems, in particular in ethnic minorities [56]. Indeed, only a small part of the screened-positive children received specialist care and we found lower service use in ethnic minority children than in ethnic majority children. However, we must keep in mind that the use of precise numerical estimates of rates of service contact to determine service need can obscure the fact that not all parents perceive emotional and behavioural problems in their child as a problem for which treatment is needed. A low service use in this group is not a good estimation of unmet need for care, as perceived need for care in this study is $23 \%$ by parents and $48 \%$ by teachers [52]. There will be a 
substantial group of children left that does not need specialist care. No care or less specialised care may be more appropriate, and sometimes preferred by parents. Therefore, we should focus on the role of other sources of care as well, since it seems unlikely and undesirable that specialised mental health care can provide care to every child [7].

\section{Limitations}

For the interpretation of our results, we must take into consideration several limitations. First, the follow-up period of 24 months could be too short to detect all children that received specialist care after signalling. Ethnic minority children may enter treatment with a greater delay than ethnic majority children, whereby we missed them. A study by Laitinen-Krispijn and colleagues (1997) showed that the predictive power of most forms of problem behaviour on service use was as high directly after signalling as it was 5 years later [18]. Their study showed a remarkable delay between the awareness of these problems in parents and their (adolescent) children and actually receiving care. Longitudinal research is needed to examine the processes for different ethnic groups of entry into and on-going contact with specialist services. Second, the Psychiatric Case Register does not provide information on alternative sources of care outside the specialist mental health system, such as care provided by schools, general practitioners and youth welfare. The case register does not include psychiatric services provided by the private sector, either. However, the exclusion of private psychiatry or psychotherapy practices is unlikely to affect our conclusions because in the Netherlands these services form only a relatively small part of the outpatient mental health care. Third, we used the SDQ to assess emotional and behavioural problems. A high SDQ score (>P90) only indicates a possible need for care, since the SDQ discerns a spectrum of emotional and behavioural problems in children, including minor problems that do not require specialist care. The cut-off of $>$ P90 includes both subclinical and clinical levels of problem behaviour. Finally, there was selective nonresponse. Non-response was higher among female children, among children living in a family composition other than both biological parents, and among children with a low severity perception according to their parents and teachers. Therefore, it is possible that these groups were underrepresented in our population. However, effect sizes were small $\left(\eta^{2}\right.$ between 0.005 and 0.022$)$ and analyses were corrected for these variables; we therefore expect that the selective non-reponse did not influence our outcomes. 


\section{Conclusion}

Already in the youngest school-aged children (5-8 years old), ethnic differences in the use of specialist mental health care are present. These differences could not be explained by ethnic variations in socioeconomic position or in parental perceived severity. Future research should explore other underlying mechanisms that can explain the ethnic differences and investigate if the lower specialist care use in ethnic minority groups is linked to an unmet need for care. Further, since perceived severity by teachers was an important predictor of health care use, teachers may be especially helpful in advising child health professionals in the process of identifying children that need (professional) care. 


\section{References}

1. Weitzman, C.C. and J.M. Leventhal, Screening for behavioral health problems in primary care. Current Opinion in Pediatrics, 2006. 18(6): p. 641-648.

2. Licence, K., Promoting and protecting the health of children and young people. Child Care Health and Development, 2004. 30(6): p. 623-635.

3. Champion, L.A., G. Goodall, and M. Rutter, Behavior Problems In Childhood And Stressors In Early Adult Life 1: A 20-Year Follow-up of London school-children. Psychological Medicine, 1995. 25(2): p. 231-246.

4. Hofstra, M.B., J. Van Der Ende, and F.C. Verhulst, Continuity and change of psychopathology from childhood into adulthood: A 14-year follow-up study. Journal of the American Academy of Child and Adolescent Psychiatry, 2000. 39(7): p. 850-858.

5. Belfer, M.L., Critical review of world policies for mental healthcare for children and adolescents. Current Opinion in Psychiatry, 2007. 20(4): p. 349-352.

6. WHO, Mental Health Atlas 2005, W.H. Organization, Editor. 2005: Geneva.

7. Ford, T., Practitioner Review: How can epidemiology help us plan and deliver effective child and adolescent mental health services? Journal of Child Psychology and Psychiatry, 2008. 49(9): $\mathrm{p}$. 900-914.

8. Sayal, K., Annotation: Pathways to care for children with mental health problems. Journal of Child Psychology and Psychiatry, 2006. 47(7): p. 649-659.

9. Leaf, P.J., et al., Mental health service use in the community and schools: Results from the fourcommunity MECA study. Journal of the American Academy of Child and Adolescent Psychiatry, 1996. 35(7): p. 889-897.

10. Angold, A., et al., Perceived parental burden and service use for child and adolescent psychiatric disorders. American Journal of Public Health, 1998. 88(1): p. 75-80.

11. Kataoka, S.H., L. Zhang, and K.B. Wells, Unmet need for mental health care among US children: Variation by ethnicity and insurance status. American Journal of Psychiatry, 2002. 159(9): p. 1548-1555.

12. Roberts, R.E., C.C. Attkisson, and A. Rosenblatt, Prevalence of psychopathology among children and adolescents. American Journal of Psychiatry, 1998. 155(6): p. 715-725. 
13. Verhulst, F.C. and J. Ende van der, Factors associated with child mental health service use in the community. Journal of the American Academy of Child and Adolescent Psychiatry, 1997. 36(7): p. 901-909.

14. Flores, G. and R.E.S. Comm Pediat, Technical Report-Racial and Ethnic Disparities in the Health and Health Care of Children. Pediatrics, 2010. 125(4): p. E979-E1020.

15. Derks, E.M., J.J. Hudziak, and D.I. Boomsma, Why more boys than girls with ADHD receive treatment: A study of Dutch twins. Twin Research and Human Genetics, 2007. 10(5): p. 765-770.

16. Kopp, S., K.B. Kelly, and C. Gillberg, Girls With Social and/or Attention Deficits: A Descriptive Study of 100 Clinic Attenders. Journal of Attention Disorders, 2010. 14(2): p. 167-181.

17. Posserud, M.B. and A.J. Lundervold, Mental Health Services Use Predicted by Number of Mental Health Problems and Gender in a Total Population Study. Scientific World Journal, 2013.

18. Laitinen-Krispijn, S., et al., Predicting adolescent mental health service use in a prospective record-linkage study. Journal of the American Academy of Child and Adolescent Psychiatry, 1999. 38(9): p. 1073-1080.

19. Gunther, N., et al., Childhood social and early developmental factors associated with mental health service use. Social Psychiatry and Psychiatric Epidemiology, 2003. 38(3): p. 101-108.

20. Jensen, P.S., L. Bloedau, and H. Davis, Children at risk 2. Risk-factors and clinic utilization. Journal of the American Academy of Child and Adolescent Psychiatry, 1990. 29(5): p. 804-812.

21. Koot, H.M. and F.C. Verhulst, Prediction of childrens referral to mental-health and specialeducation services from earlier adjustment. Journal of Child Psychology and Psychiatry and Allied Disciplines, 1992. 33(4): p. 717-729.

22. Cunningham, P.J. and M.P. Freiman, Determinants of ambulatory mental health services use for school-age children and adolescents. Health Services Research, 1996. 31(4): p. 409-427.

23. Staghezza-Jaramillo, B., et al., Mental health service utilization among Puerto Rican children ages 4 through 16. Journal of Child and Family studies, 1997. 4(4): p. 399-418.

24. Farmer, E.M.Z., et al., Use, persistence, and intensity: Patterns of care for children's mental health across one year. Community Mental Health Journal, 1999. 35(1): p. 31-46. 
25. Sayal, K., The role of parental burden in child mental health service use: Longitudinal study. Journal of the American Academy of Child and Adolescent Psychiatry, 2004. 43(11): p. 13281333.

26. Zwaanswijk, M., et al., Help seeking for emotional and behavioural problems in children and adolescents - A review of recent literature. European Child \& Adolescent Psychiatry, 2003. 12(4): p. $153-161$.

27. Zwaanswijk, M., et al., Change in children's emotional and behavioural problems over a one-year period - Associations with parental problem recognition and service use. European Child \& Adolescent Psychiatry, 2006. 15(3): p. 127-131.

28. Berk, L.E., Child Development. 5th ed. 2000, Massachusetts: Ally and Bacon.

29. De Los Reyes, A. and A.E. Kazdin, Informant discrepancies in the assessment of childhood psychopathology: A critical review, theoretical framework, and recommendations for further study. Psychological Bulletin, 2005. 131(4): p. 483-509.

30. Stevens, G., et al., Parent, teacher and self-reported problem behavior in The Netherlands Comparing Moroccan immigrant with Dutch and with Turkish immigrant children and adolescents. Social Psychiatry and Psychiatric Epidemiology, 2003. 38(10): p. 576-585.

31. Sonuga-Barke, E.J.S., et al., Interethnic bias in teachers ratings of childhood hyperactivity. British Journal of Developmental Psychology, 1993. 11: p. 187-200.

32. Epstein, J.N., et al., The role of children's ethnicity in the relationship between teacher ratings of attention-deficit/hyperactivity disorder and observed classroom behavior. Journal of Consulting and Clinical Psychology, 2005. 73(3): p. 424-434.

33. Goodman, R., The strengths and difficulties questionnaire: A research note. Journal of Child Psychology and Psychiatry and Allied Disciplines, 1997. 38(5): p. 581-586.

34. Goodman, R., Psychometric properties of the Strengths and Difficulties Questionnaire. J Am Acad Child Adolesc Psychiatry, 2001(40): p. 30-40.

35. Mieloo, C.L., et al., Validity and Reliability of the Strengths and Difficulties Questionnaire in 5-6 Year Olds: Differences by Gender or by Parental Education? . Plos-One, 2012. 7(5).

36. van Widenfelt, B.M., et al., Dutch version of the Strengths and Difficulties Questionnaire (SDQ). Eur Child Adolesc Psychiatry, 2003(12): p. 281-289. 
37. Goodman, R., et al., Using the Strengths and Difficulties Questionnaire (SDQ) to screen for child psychiatric disorders in a community sample. British Journal of Psychiatry, 2000. 177: p. 534-539.

38. Goodman, R., The extended version of the Strengths and Difficulties Questionnaire as a guide to child psychiatric caseness and consequent burden. Journal of Child Psychology and Psychiatry, 1999. 40: p. 791-801.

39. CBS, Statistical Yearbook of the Netherlands. 2002, Voorburg/Heerlen.

40. Ten Horn, G.G.M.M., Definitions and classifications: introduction, in Psychiatric Case Registers in Public Health: A Worldwide Inventory 1960-1985, G.G.M.M. Ten Horn, et al., Editors. 1986, Elsevier: Amsterdam. p. 170-174.

41. Wierdsma, A.I., et al., Case registers in psychiatry: do they still have a role for research and service monitoring? Current Opinion in Psychiatry, 2008. 21(4): p. 379-384.

42. Newcombe, H.B., Handbook of Record Linkage: Methods for Health and Statistical Studies, Administration, and Business. 1988, New York: Oxford University Press.

43. Saxena, S., J. Eliahoo, and A. Majeed, Socioeconomic and ethnic group differences in self reported health status and use of health services by children and young people in England: cross sectional study. British Medical Journal, 2002. 325(7363): p. 520-523.

44. Brown, J.D., et al., Receiving advice about child mental health from a primary care provider African American and Hispanic parent attitudes. Medical Care, 2007. 45(11): p. 1076-1082.

45. Bussing, R., et al., Exploring help-seeking for ADHD symptoms: A mixed-methods approach. Harvard Review of Psychiatry, 2005. 13(2): p. 85-101.

46. Costello, E.J., H. Egger, and A. Angold, 10-year research update review: The epidemiology of child and adolescent psychiatric disorders: I. Methods and public health burden. Journal of the American Academy of Child and Adolescent Psychiatry, 2005. 44(10): p. 972-986.

47. Owens, P.L., et al., Barriers to children's mental health services. Journal of the American Academy of Child and Adolescent Psychiatry, 2002. 41(6).

48. Ford, T., et al., Predictors of Service Use for Mental Health Problems Among British Schoolchildren. Child and Adolescent Mental Health, 2008. 13(1): p. 32-40.

49. Sourander, A., et al., Child and adolescent mental health service use in Finland. Social Psychiatry and Psychiatric Epidemiology, 2001. 36(6): p. 294-298. 
50. Cohen, P., et al., An epidemiological study of disorders in late childhood and adolescence, I: ageand gender-specific prevalence. Journal of Child Psychology and Psychiatry, 1993. 34: p. 851867.

51. Farmer, E.M.Z., et al., Pathways into and through mental health services for children and adolescents. Psychiatric Services, 2003. 54(1): p. 60-66.

52. Bevaart, F., et al., Ethnic differences in problem perception and perceived need for care for young children with problem behaviour. Journal of Child Psychology and Psychiatry and Allied Disciplines, 2012. 53(10): p. 1063-1071.

53. Sayal, K., E. Taylor, and J. Beecham, Parental perception of problems and mental health service use for hyperactivity. Journal of the American Academy of Child and Adolescent Psychiatry, 2003. 42(12): p. 1410-1414.

54. Teagle, S.E., Parental Problem Recognition and Child Mental Health Service Use. Mental Health Services Research, 2002. 4(4): p. 257-266.

55. Sourander, A., et al., Have there been changes in children's psychiatric symptoms and mental health service use? A 10-year comparison from Finland. Journal of the American Academy of Child and Adolescent Psychiatry, 2004. 43(9): p. 1134-1145.

56. Sheldrick, R.C., S. Merchant, and E.C. Perrin, Identification of Developmental-Behavioral Problems in Primary Care: A Systematic Review. Pediatrics, 2010. 128: p. 356-363. 